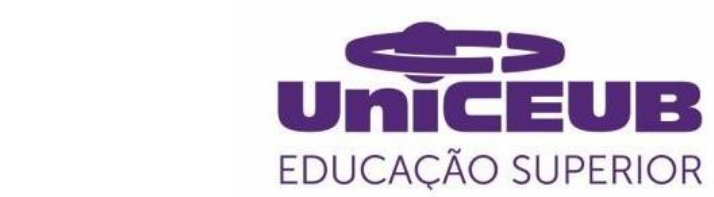

CENTRO UNIVERSITÁRIO DE BRASÍLIA - UnICEUB

PROGRAMA DE INICIAÇÃO CIENTÍFICA

KESSLER REZENDE OLIVEIRA

ROBSON ARAÚJO DE OLIVEIRA

AUTOMAÇÃO DE CONTROLE DO NÍVEL DA ÁGUA PARA SISTEMA AQUAPÔNICO DOMICILIAR

BRASÍLIA

2020

p. 1 


\section{Untce $\operatorname{Ba}$ \\ EDUCAÇÃO SUPERIOR}

KESSLER REZENDE OLIVEIRA

ROBSON ARAÚJO DE OLIVEIRA

\section{AUTOMAÇÃO DE CONTROLE DO NÍVEL DA ÁGUA PARA SISTEMA AQUAPÔNICO DOMICILIAR}

Relatório final de pesquisa de Iniciação Científica apresentado à Assessoria de PósGraduação e Pesquisa.

Eduardo José Ribeiro de Castro

\section{BRASÍLIA}

2020 


\section{DEDICATÓRIA}

Dedicamos este trabalho acadêmico ao nosso único Senhor e Salvador Jesus Cristo o próprio Deus a quem devemos toda a honra e toda a glória aqui na Terra e no Céu para todo o sempre. Amém.

p. 3 


\section{AGRADECIMENTOS}

Agradecemos ao Professor e Mestre Eduardo José Ribeiro de Castro por acreditar na relevância da nossa pesquissa e por ter contribuído de forma significativa no processo de nossa formação profissional no curso de Análise e Desenvolvimento de Sistemas do UniCEUB.

p. 4 


\section{RESUMO}

A aquaponia é uma modalidade de produção de alimentos que une a piscicultura para cultivo de peixes e a hidroponia para fertilização de plantas sem o uso de solo com o suporte de bactérias aeróbicas e anaeróbicas em um sistema de circulação fechada em pequena escala. O termo aquaponia é derivado da combinação das palavras "aquicultura" para produção de organismos aquáticos e 'hidroponia' para produção de plantas sem solo. A aquaponia preconiza a reutilização da água em sistema de circulação fechado, reduzindo desperdícios e diminuindo a liberação de efluentes tóxicos no meio ambiente. O volume de água necessário para um sistema de aquaponia é muito baixo quando comparado aos sistemas tradicionais de agricultura e aquicultura separadamente. A água do sistema de aquaponia não precisa ser trocada periodicamente, porque os compostos sais, ácidos e bases da ração dos peixes são tratados bioquimicamente por bactérias e posteriormrnte convertidos em nutrientes absorvíveis pelos vegetais hidropônicos, bastando apenas realizar uma reposição automática da água perdida no processo de evaporação. O projeto de pesquisa desenvolveu um sistema automatizado de controle de nível da água na plataforma aquapônica domiciliar utilizando um dispositivo embarcado de Internet das Coisas, utilizando a liguagem de programação C++ e o sistema operacional Linux.

Palavras-Chave: Aquaponia. Hiprodonia. Psicicultura. Internet das Coisas. 


\section{LISTAS DE FIGURAS}

Figura 1

p. 9

Figura 2

p. 10

Figura 3

p. 11

Figura 4

p. 11

Figura 5

p. 12

Figura 6

p. 14

Figura 7

p. 22

Figura 8

p. 22

Figura 9

p. 23 


\section{SUMÁRIO}

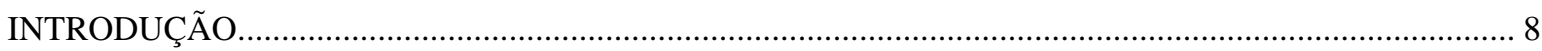

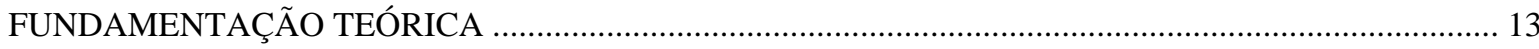

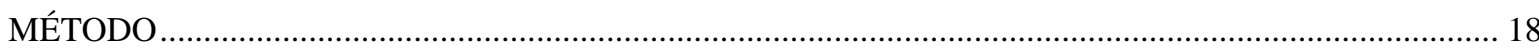

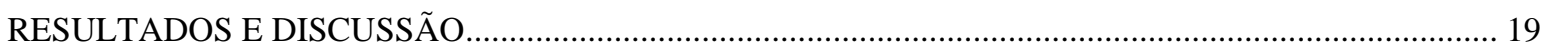

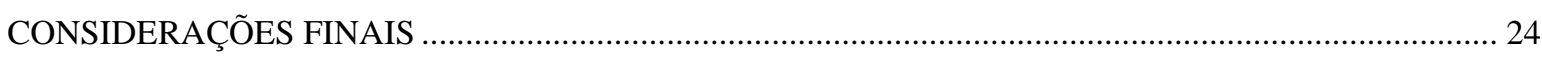

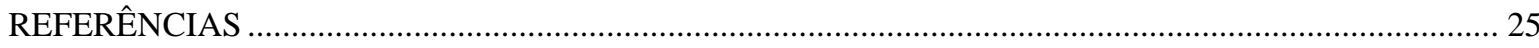

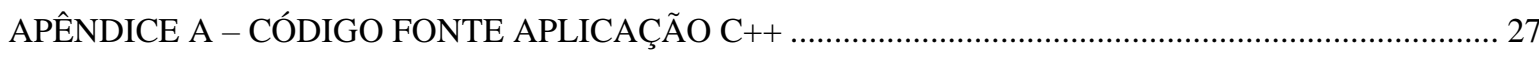




\section{INTRODUÇÃO}

A aquaponia é uma modalidade de produção de alimentos que une a Piscicultura (cultivo de peixes) e a Hidroponia (fertilização de plantas sem o uso de solo) com o suporte de bactérias em um sistema de circulação fechada em pequena escala ${ }^{12}$.

O termo aquaponia é derivado da combinação das palavras "aquicultura" (produção de organismos aquáticos) e 'hidroponia' (produção de plantas sem solo). A aquaponia preconiza a reutilização total da água, evitando seu desperdício e diminuindo drasticamente, ou até eliminando, a liberação do efluente no meio ambiente. O volume de água necessário para um sistema de aquaponia é muito baixo quando comparado aos sistemas tradicionais de agricultura e aquicultura. A água do Sistema de Aquaponia não precisa ser trocada periodicamente, pois, sais provenientes dos efluentes tratados são transformados em nutrientes que serão gradativamente absorvidos pelos vegetais hidropônicos, bastando apenas realizar periodicamente a reposição periódica da água que evapora.

Em um sistema de aquaponia os dejetos das urinas e fezes dos peixes (amônia e amônio) devem ser retirados do tanque dos peixes através da constante circulação da água e através do processo de decantação e filtragem destes elementos eles são devolvidos para o tanque dos peixes livre de contaminantes. A aquaponia é mais eficiente na reutilização água dos peixes contendo efluentes tratados bioquimicamente para o fornecimento de nutrientes para os vegetais hidropônicos ${ }^{3}$.

Desta forma, as toxinas, consideradas dejetos indesejáveis produzidas pelo tanque dos peixes se transforma em insumo para o tanque dos hidropônicos que por sua vez a água é filtrada e devolvida para o tanque peixes, sem a necessidade de se gastar com manutenção de filtros, recurso indispensável em sistemas tradicionais de piscicultura, bem como reduz a necessidade de gasto adicional com fertilizantes na produção de hidropônicos, elevando o potencial de produção de alimentos com redução de custos, pois, cria um sistema de colaboração mútua entre a produção de peixes e de vegetais hidropônicos. Apesar de haver um equilíbrio dinâmico na redução de toxinas no sistema de

\footnotetext{
1 EMBRAPA (2015a)

2 PATTILLO e ALLEN (2017a)

${ }^{3}$ EMBRAPA (2015b)
} 
Aquaponia é necessário controlar também os níveis de $\mathrm{PH}$, temperatura, nível de água dos tanques, potencial de oxidação e redução, nível de oxigênio dissolvido e irrigação dos hidropônicos com base na umidade relativa do ar utilizando ciclos manuais de medições (sensores) e intervenções diárias (despejo de soluções: oxidantes, redutoras, germicidas e nutrientes com de reposição de água perdida por evaporação ou vazamento na plataforma ${ }^{4}$.

Os processos manuais de operação dos sistemas de aquaponia convencionais são descritos conforme a representação do modelo cíclico abaixo:

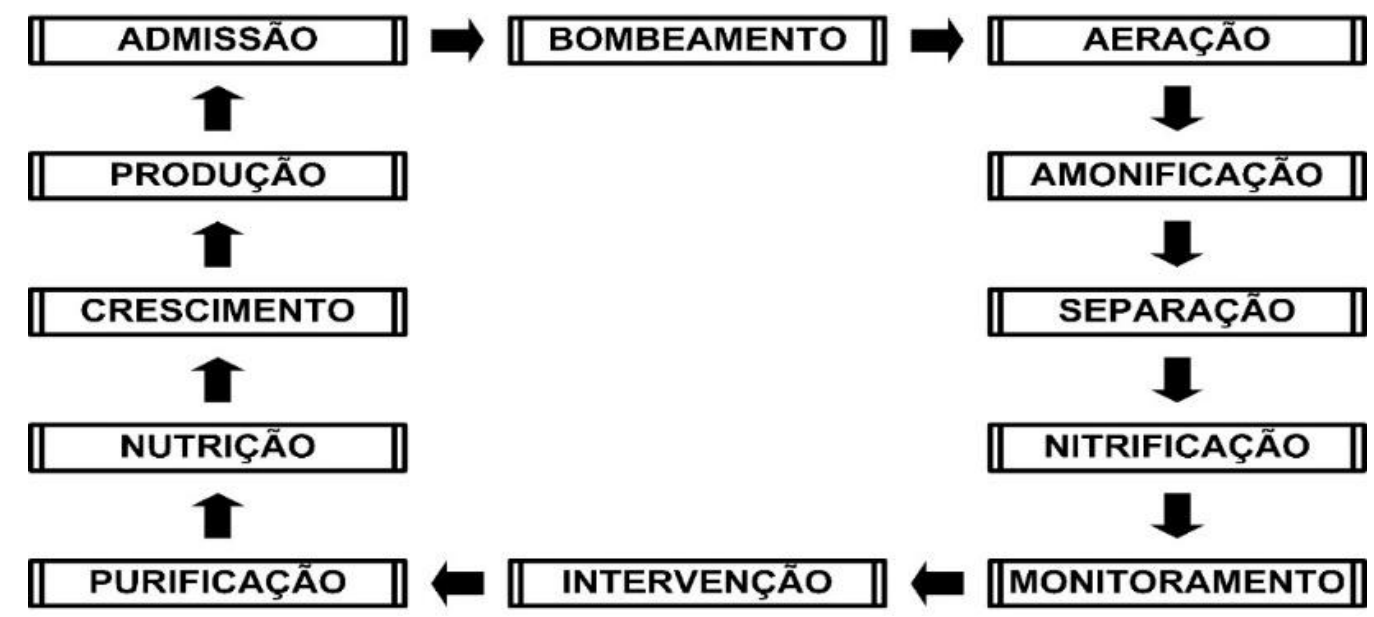

Figura 1 - Processos manuais sistemas aquaponia convencionais EMBRAPA (2016).

\section{OBJETIVO GERAL DO PROGRAMA DE INICIAÇÃO CIENTÍFICA:}

Construir um módulo para automação de controle do nível da água para sistema aquapônico domiciliar.

A Central de Comando e Controle utilizará sensores e microcontroladores para realizar o monitoramento dos níveis de água na plataforma conforme o medelo esquemático abaixo:

${ }^{4}$ EMBRAPA (2017) 


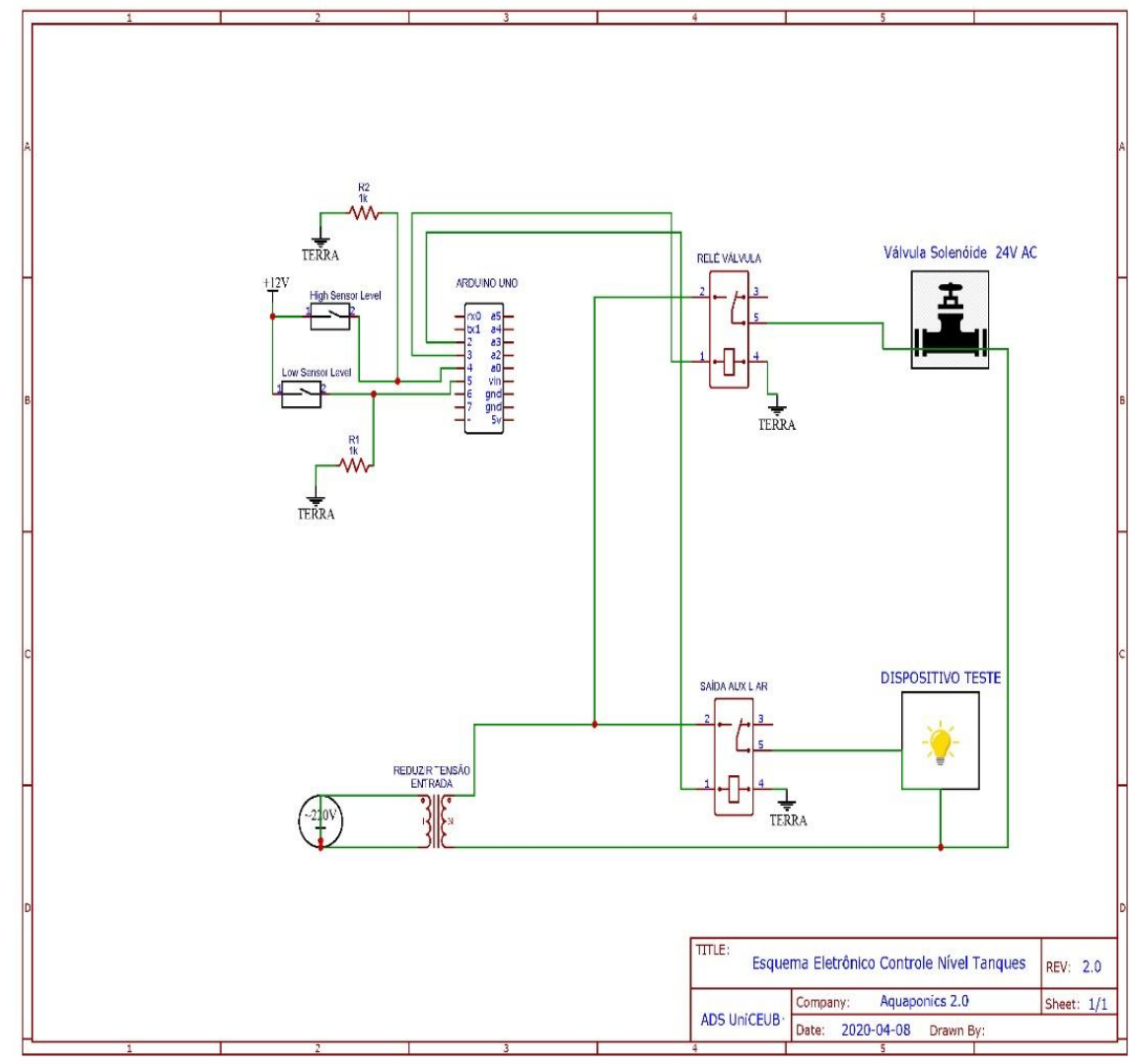

Figura 2 - Quadro comando controle nível água aquaponia domiciliar.

\section{OBJETIVOS ESPECÍFICOS DO PROGRAMA DE INICIAÇÃO CIENTÍFICA:}

Identificar os requisitos de negócio para criação de uma plataforma de automação dos sistemas de Aquaponia para produção de peixes e plantas em pequena escala; e

Projetar as plataformas de hardware, software e rede para o funcionamento local do módulo para o controle do nível da água no sistema Aquapônico domiciliar. 


\section{ANÁLISE DOS REQUISITOS}

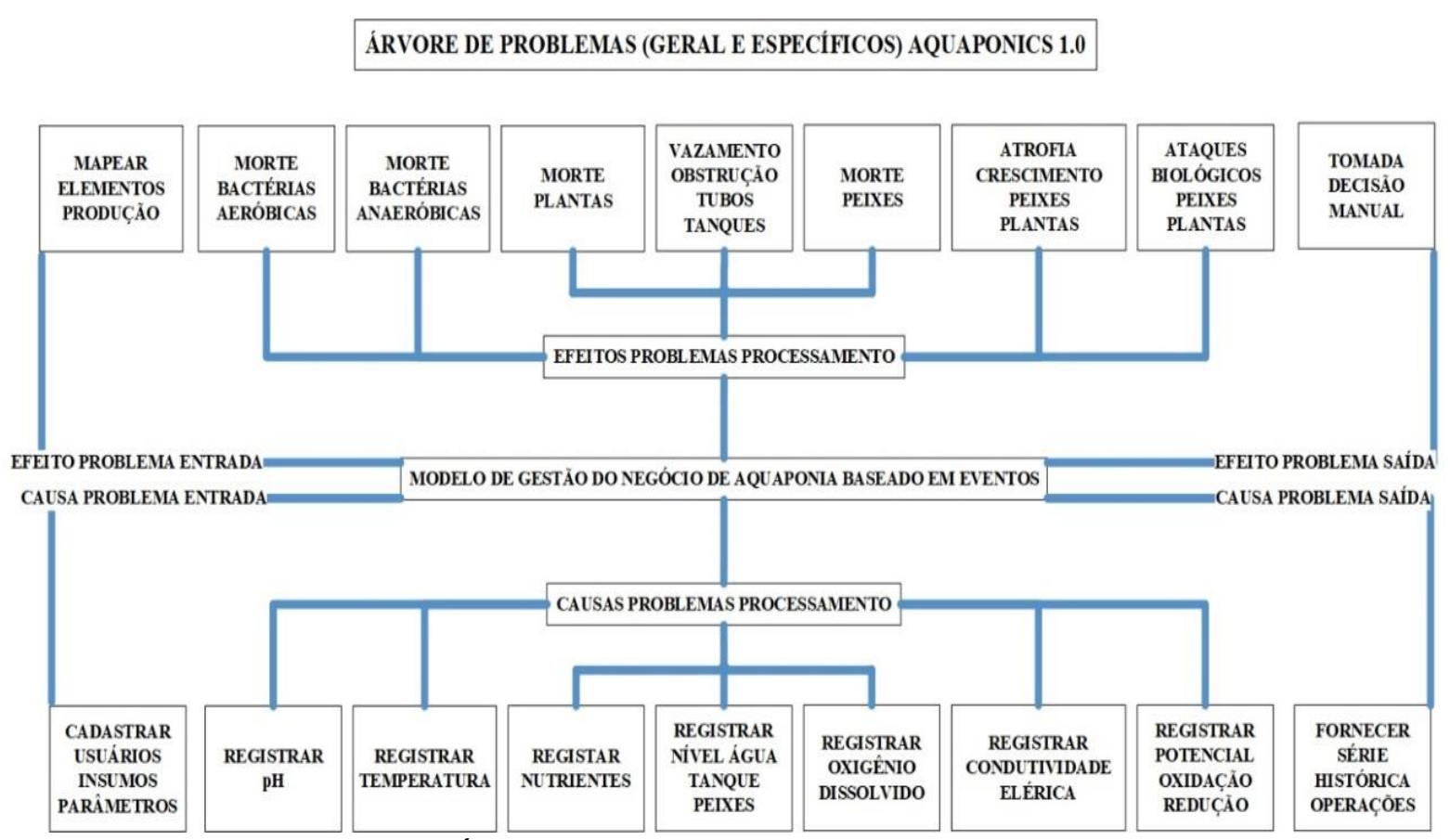

Figura 3 - Árvore de problemas da plataforma de aquaponia.

ARVORE DE OBJETIVOS (GERAL E ESPECIFICOS) AQUAPONICS 1.0

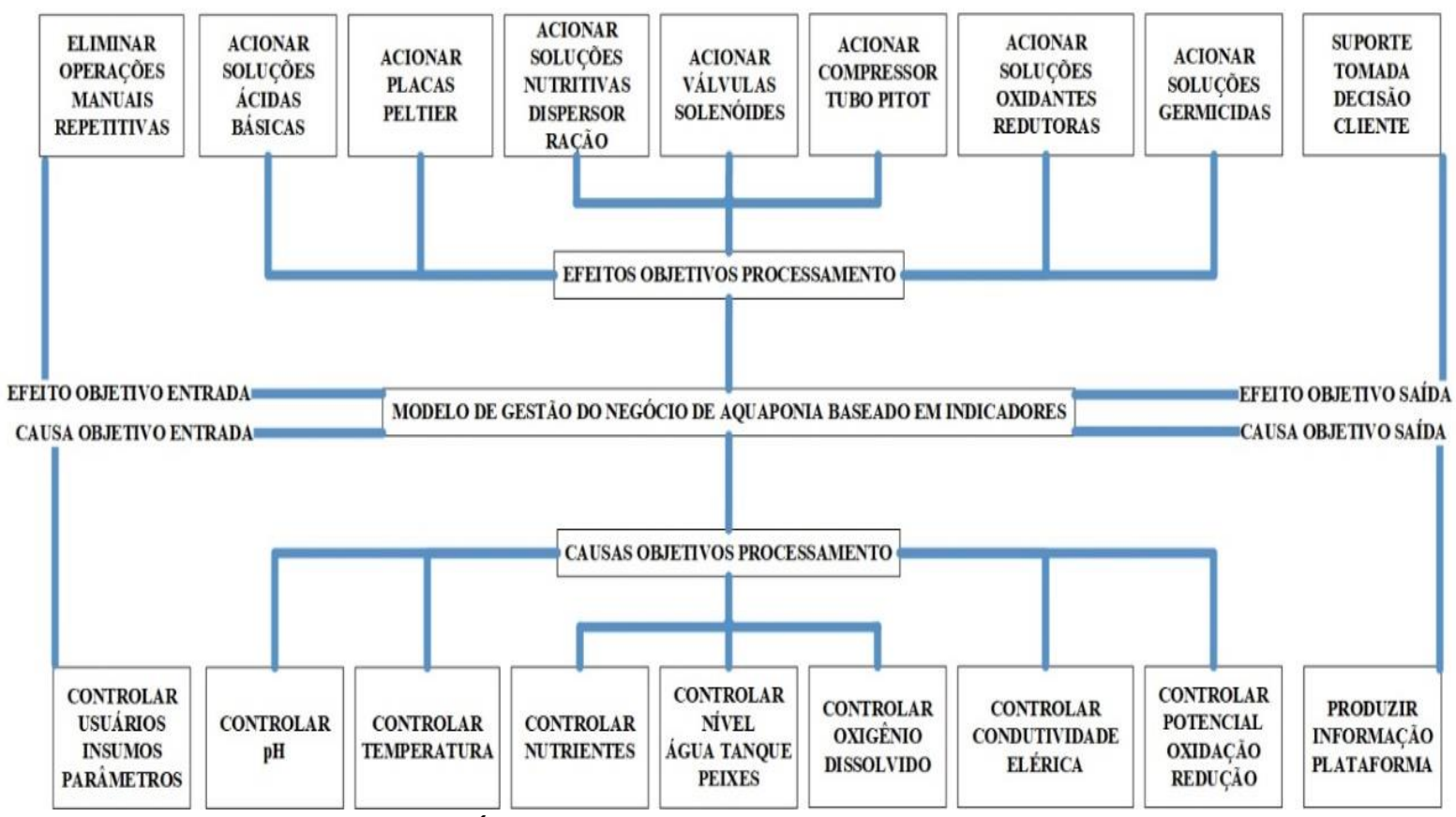

Figura 4 - Árvore de soluções da plataforma de aquaponia. 


\section{IDENTIFICAÇÃO DAS FUNCIONALIDADES}

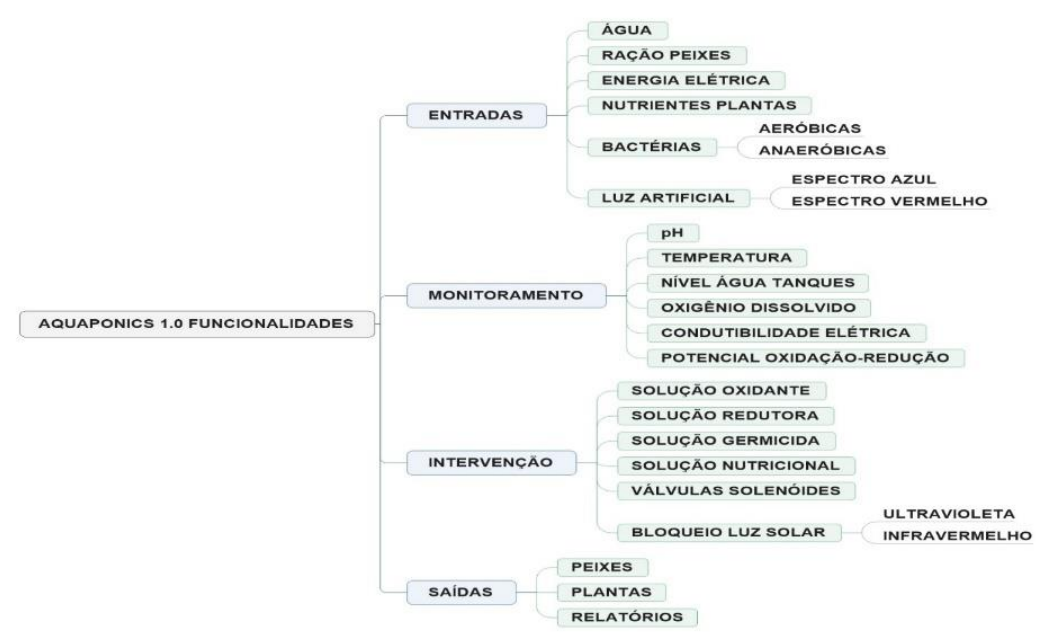

Figura 5 - Funcionalidades da solução da plataforma de aquaponia.

\section{Justificativa}

A criação de peixes associada ao cultivo de hortaliças, chamada de aquaponia, pode economizar até $90 \%$ de água em relação à agricultura convencional e ainda eliminar completamente a liberação de efluentes no meio ambiente, pois trata-se de um sistema fechado, diferentemente das criações convencionais. Os pesquisadores da Unidade Embrapa em Tabuleiros Costeiros no Estado de Sergipe construíram sistemas aquapnicos de diferentes tamanhos para produção doméstica e exporação comercial em larga escala, no entanto, todas as soluções são baseadas em processos manuais de monitoramento, cálculo e intervenção no ambiente de produção de aquaponia ao menos 3 vezes por dia em ciclos de 8 em 8 horas, demandando atenção e conhecimento técnico para o correto funcionamento da plataforma aquapônica instalada 5 . Considerando as premissas éticas e bio-sanitárias do projeto de iniciação científica do UniCEUB não serão utilizados peixes nos experimentos, porque o processso de nitrificação alvo da pesquisa será induzido pela adição manual de uréia sintética na plataforma para emular as reações químicas de contaminação da água por decomposição de matéria orgânica e transformação da amônia $\left(\mathrm{NH}_{4}\right)$ e do amônio $\left(\mathrm{NH}_{3}\right)$ dissolvidos na água em substratos nitrogenados nutritivos dos tipos nitratos $\left(\mathrm{NO}_{2}\right)$ e nitritos $\left(\mathrm{NO}_{3}\right)$ para serem utilizados pelas plantas.

${ }^{5}$ EMBRAPA (2016) 


\section{FUNDAMENTAÇÃO TEÓRICA}

Segundo EMBRAPA (2015b), o fornecimento de ração aos peixes é a entrada de insumo mais importante num sistema aquapônico. Os peixes se alimentam da ração e produzem excretas que são convertidas nos nutrientes que, posteriormente, serão absorvidos pelas plantas. Na aquaponia, há um fluxo contínuo de nutrientes entre diferentes organismos vivos que estão relacionados por meio de ciclos biológicos naturais, notadamente a nitrificação promovida por bactérias. As bactérias nitrificantes dos gêneros nitrosomonas e nitrobacter são responsáveis pela conversão da amônia (NH3) em nitrito (NO2) e este em nitrato (NO3), transformando substâncias tóxicas produzidas pelos peixes em nutrientes assimiláveis pelas plantas. Ao consumir esses nutrientes as plantas, juntamente com as bactérias, desempenham papel importante na filtragem biológica da água, garantindo sua condição adequada para o desenvolvimento normal dos peixes.

Para EMBRAPA (2015b), o nitrogênio é o nutriente requerido pelas plantas em maior quantidade e o nitrato é a forma preferida de absorção, a compreensão e o manejo adequado das colônias de bactérias é de fundamental importância na aquaponia. O surgimento dessas bactérias num sistema de aquaponia se dá de forma natural num ambiente chamado de filtro biológico, porém pode ser estimulado pela introdução de água trazida de outro local onde é conhecida sua presença. Já o pH é um dos pontos mais críticos e que requer muita atenção dentro de um sistema de aquaponia. Pelo fato da aquaponia envolver num mesmo corpo d'água três organismos muito distintos (peixes, plantas e bactérias) é de fundamental importância conhecer as necessidades de cada um deles para que $\mathrm{o} \mathrm{pH}$ da água seja mantido numa faixa que atenda a todos satisfatoriamente. As bactérias nitrificantes são predominantemente aeróbicas e têm o pH ótimo no intervalo entre 7,0 e 8,0 . Por outro lado, a maioria das plantas cultivadas em hidroponia cresce melhor em pH entre 5,5 e 6,5. Já para a maioria das espécies peixes de água doce de interesse econômico e que podem ser utilizados num sistema aquapônico, o pH ideal encontra-se entre 7,0 e 9,0. Com isso, recomenda-se que o pH da água seja mantido entre 6,5 e 7,0 para atender satisfatoriamente a todos os componentes biológicos presentes num sistema aquapônico. 


\section{Componentes do Sistema de Aquaponia}

Diversos centros de pesquisa e desenvolvimento nas áreas de agropecuária e pesca vem aprofundando estudos sobre a produção orgânica de proteína animal e vegetal em pequena escala para garantir a segurança alimentar da população em casos de guerra ou de desastres naturais em larga escala, dentre eles temos como paradigma o modelo de Aquaponia da Universidade Estadual de lowa (PATTILLO, 2017).

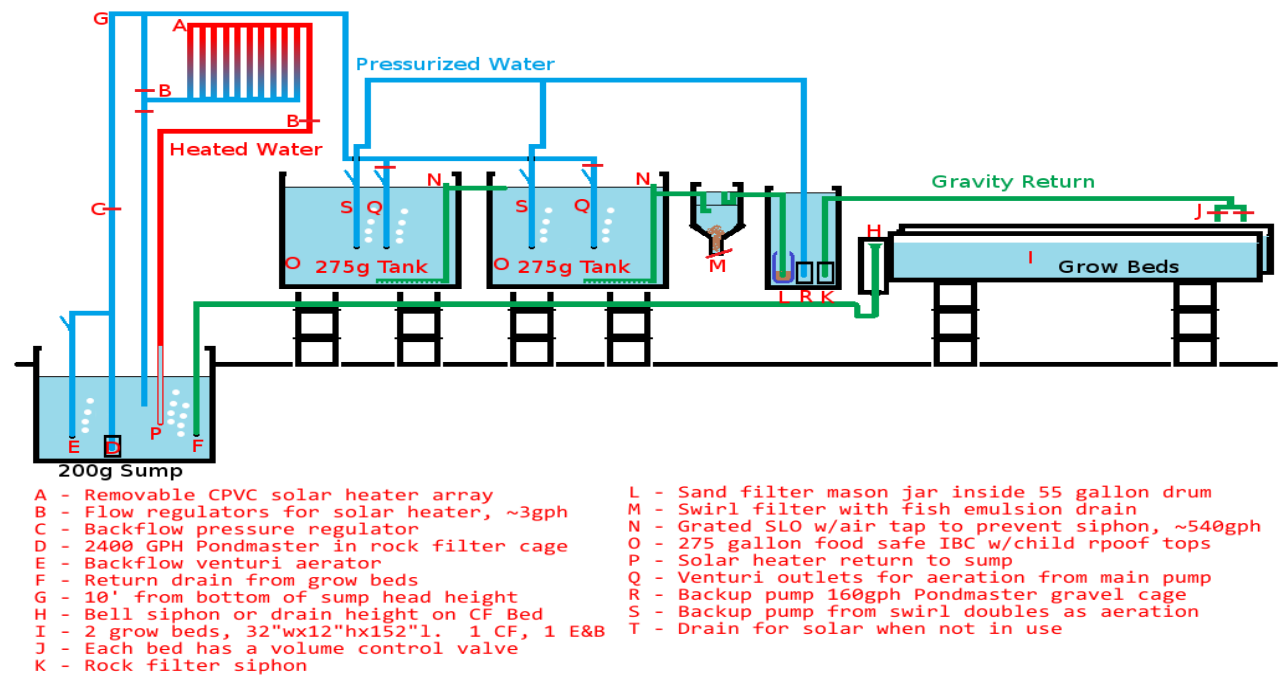

Figura 6 - Plataforam de aquaponia padrão da Universidade de IOWA

Tanque dos peixes - O ambiente de criação dos peixes dentro de um sistema aquapônico é geralmente representado por um ou vários tanques nos mais diversos formatos e volumes, podendo variar de poucos litros a vários metros cúbicos e ser feito de vários tipos de materiais, desde que sejam resistentes e duráveis (EMBRAPA, 2015b). Em sistemas aquapônicos de pequeno porte, nos quais são utilizadas densidades de estocagem de peixes inferiores a $10 \mathrm{~kg} / \mathrm{m} 3$ são utilizados tanques ou caixas entre $100 \mathrm{~L}$ e $1.000 \mathrm{~L}$.

O fluxo de água que passa pelo tanque de criação de peixes deve levar em conta fatores como a velocidade da água e a taxa de renovação. A velocidade da água dentro do tanque não pode ser tão rápida a ponto de exigir esforço natatório demasiado dos peixes e, por outro lado, a velocidade da água precisar ser tal que possa auxiliar na retirada dos dejetos produzidos pelos peixes e evitar seu acúmulo dentro do tanque. 
Decantadores - Sistemas aquapônicos com baixa biomassa de peixes, abaixo de 5 kg/m3, produzem poucos resíduos sólidos. Estes, por sua vez, são normalmente capturados e degradados dentro do próprio filtro biológico. Entretanto, o emprego de densidades mais elevadas, principalmente acima de $10 \mathrm{~kg} / \mathrm{m} 3$, exige o uso de artifícios que permitam a retirada constante dos resíduos sólidos. Resíduos mais densos podem ser separados por algum aparato de filtração que promova sua sedimentação.

Sistema de aeração - Na aquaponia, a aeração é exigida não apenas pelos peixes, mas também pelas raízes das plantas e pelas bactérias nitrificantes do filtro biológico. Em clima tropical, a quantidade de oxigênio dissolvido na água deve ser sempre superior a 3 $\mathrm{mg} / \mathrm{L}$ e pode ser mantida por compressores ou sopradores de ar (Figura 6). A aeração deve ser fornecida diretamente na água do tanque de criação dos peixes e também no ambiente de cultivo de vegetais quando se tratar do ambiente flutuante, conforme será apresentado posteriormente.

Ambientes de cultivo de vegetais - São vários os tipos de ambientes (ou substratos) utilizados para o cultivo de vegetais em aquaponia, cada um com suas particularidades e variações, vantagens e desvantagens. Entre os mais comuns, temos o ambiente de cultivo em cascalho, ambiente flutuante e ambiente de cultivo em canaletas.

As melhores práticas internacionais em Aquaponia apresentam muitas vantagens em relação aos modelos de aquicultura e hidroponia convencionais funcionando separadamente, dentre as quais podemos destacar:

- Redução nas áreas de plantio e manejo;

- Redução no consumo de água e energia elétrica;

- Redução no consumo de fertilizantes e defensivos;

- Produção anual em condições climáticas extremas; e

- Ausência de solos normalmente infestados de vetores, parasitas e ervas daninhas. 
Conceito de Internet das Coisas (IdC)

Segundo Santos et. al (2017) a Internet das Coisas nada mais é que uma extensão da rede para comunicação de dados englobando dispositivos com capacidade de processamento reduzido. A conexão com a Internet permite tanto controlar remotamente os objetos IdC quanto conectá-los com provedores de serviços externos.

No contexto operacional da IdC uma unidade básica de hardware deverá apresentar as funcionalidades abaixo (RUIZ et al. 2004 e LOUREIRO et al. 2003):

- Unidade de processamento;

- Unidade de memória;

- Unidade de comunicação;

- Unidade de sensor; e

- Unidade de atuador.

Segundo Santos et. al (2017) os dispositivos IdC são denominados objetos inteligentes porque que estabelecerem comunicações com sensores, atuadores e equipamentos de redes para transmissão de dados.

Blocos Básicos de Construção da IdC

A Internet da Coisas é uma plataforma computacional que combina diversas tecnologias para integrar objetos inteligentes do ambiente físico ao mundo virtual (Bruno et. al, 2017), conforme o modelo conceitual abaixo:

10 Bloco IdC (Identificação): identifica os objetos inteligentes que demandam conexão com a Internet utilizando as tecnologias RFID (Radio-Frequency Identification), NFC (Near Field Communication) e padrões de endereçamento IP (Internet Protocol).

2을 Bloco IdC (Sensores/Atuadores): sensores coletam informações sobre o contexto onde os objetos se encontram e, em seguida, armazenam/encaminham esses dados para data warehouse, clouds ou centros de armazenamento. Atuadores podem manipular o ambiente ou reagir de acordo com os dados lidos. 
3으이oco IdC (Comunicação): diz respeito às diversas técnicas usadas para conectar objetos inteligentes. Também desempenha papel importante no consumo de energia dos objetos sendo, portanto, um fator crítico. Algumas das tecnologias usadas são WiFi, Bluetooth e IEEE 802.15.4.

40 Bloco IdC (Computação): inclui a unidade de processamento como placas microcontroladoras responsáveis por executar algoritmos locais nos objetos inteligentes.

5응oco IdC (Serviços): Tarefas de Identificação, responsáveis por mapear Entidades Físicas (EF) dos usuários em Entidades Virtuais (EV) registors de monitoramento e controle da plataforma.

60 Bloco IdC (Serviços de Agregação de Dados): coletam e processam informações obtidos pelos objetos inteligentes;

70 Bloco IdC (Serviços de Colaboração e Inteligência): agem sobre os serviços de agregação de dados para tomar decisões e reagir de modo adequado a um determinado cenário.

8응 Bloco IdC (Semântica): descoberta de conhecimento e uso eficiente dos recursos existentes a partir dos dados existentes para melhor um produto ou um serviço.

\section{Tecnologias de Hardware}

Antigamente o fator custo era limitante para adoção e desenvolvimento de objetos inteligentes (Santos et. al, 2017). Entretanto, hoje em dia é possível encontrar soluções de IdC disponíveis no mercado a um custo acessível. Produtos como o Raspberry Pi, Arduino e similares permitem desde a prototipagem até a produção final de soluções de $\operatorname{IdC}$ a baixo custo, ou seja, é possível encontrar o Raspberry Pi ao custo de US\$ 35.

\section{Sistemas Operacionais}

Segundo Santos et. al (2017) os dois principais sistemas operacionais para objetos inteligentes são o Contiki e TinyOS, além do sistema operacional Android que opera em grande parte dos smartphones e algumas variações do sistema Linux orientadas à IdC, como é o caso do Raspbian que é um SO baseado no Debian e otimizado para o hardware do Raspberry Pi. O Raspberry Pi oferece mais de 35 mil pacotes deb de software, que estão 
pré-compilados, para serem facilmente instalados no Raspberry Pi, como também o Ubuntu que também possui sua versão IdC, chamada Ubuntu Core ou Snappy. Essa versão de sistema operacional é reduzida em comparação ao Ubuntu desktop, uma vez que exige apenas $128 \mathrm{MB}$ de memória RAM e um processador de $600 \mathrm{MHz}$.

\section{MÉTODO}

Esse trabalho construiu uma plataforma de aquaponia domiciliar de baixo custo com o suporte da tecnologia de IdC num ambiente controlado sem o uso de animais.

(a) Tipificação: pesquisa qualitativa.

(b) Abordagem: Integração de Requisitos Orientados ao Negócio (IRON).

(c) Procedimento: Análise de Requisitos.

(d) Caracterização do local de pesquisa: residência dos alunos.

(e) Custeio: recursos financeiros pessoais dos alunos.

(f) Objeto de estudo: aquaponia.

(g) Delimitação do escopo da pesquisa: automação do controle de nível de água do sistema de aquaponia domiciliar.

(e) Instrumento para geração de dados: platafomra de IdC.

(f) Gerenciamento do projeto: estrutura analítica de software.

Etapas da pesquisa

- Revisão bibliográfica;

- Teste em protótipo em escala reduzida;

- Identificar critérios de seleção da solução;

- Construção de testes automatizados da solução;

- Avaliação dos impactos da solução de automação; e

- Construção da solução de automação com base nos critérios Identificados. 
Tendo em vista a compatibilidade e aderência da solução no contexto do problema, a solução deve atender alguns critérios básicos para sua efetiva aceitação e aderência devendo atender os seguintes critérios mínimos de homologação da solução :

Baixo custo de implementação;

Plataforma que utiliza linguagem de programação de alto nível;

Ambiente de simulação para construção de testes unitários no protótipo; e

Versatilidade para permitir controle automático local sem conexão com a Internet.

Hipótese de Pesquisa:

A solução de automação para aquaponia domiciliar deverá ter um custo total inferior a $\mathrm{R} \$$ 5.000, garantindo também fácil operação por parte de um usuário sem formação técnica com um baixo custo de manutenção anual.

Hipótese Nula:

A solução não é economicamente viável ou não é capaz de promover a eliminação de contaminantes sintéticos adicionados manualmente na plataforma.

\section{RESULTADOS E DISCUSSÃO}

Os resultados da hipótese da pesquisa foram alcançados e o custo global do projeto foram detalhados conforme o memorial de cálculos abaixo:

2 (duas) Bóais Eletrônicas IdC R\$ 50,00)

1 (uma) Bomba submersível (R\$ 195,00)

4 (quatro) Lixas de Ferro $n=100(R \$ 10,00)$

10 (dez) metros mangueira silicone $(R \$ 20,00)$

1 (um) 'T' Soldável em PVC de 25 mm (R\$ 5,00)

1 (uma) Válvula Solenóide Eletrônica ( $R \$ 200,00$ )

1 (um) Compressor eletromagnético ar ( $R \$ 312,00)$ 
2 (dois) Baldes de Plástico de 20 Litros (R\$ 20,00)

2 (dois) Containers IBC de 1000 Litros (R\$ 500,00)

3 (três) 'Ts' Soldáveis em PVC de 32 mm (R\$ 10,00)

3 (três) 'Ts' Soldáveis em PVC de 20 mm (R\$ 15,00)

3 (três) Abraçadeiras em Metal de 32 mm (R\$ 5,00)

2 (dois) Tambores Plásticos de 150 Litros (R\$ 100,00)

2 (dois) Litros de Solvente Tipo Removedor $(\mathrm{R} \$ 40,00)$

1 (um) Disjuntor Temporizado Digital Trilho (R\$179,00)

1 (um) Metro de Tubo para Esgoto de 75 mm (R\$ 10,00)

1 (um) Compressor Eletromagnético de 16W R\$ $(100,00)$

1 (um) Metro de Tubo para Esgoto de 100 mm (R\$ 20,00)

1 (uma) fonte regulável rebaixamento tensão (( $R \$ 100,00)$

1 (uma) Placa Microcontroladora Arduino Uno (R\$ 200,00)

6 (seis) Metros de Tudo Soldável PVC de 25 mm (R\$20,00)

6 (seis) Metros de Tudo Soldável PVC de 20 mm (R\$ 10,00)

6 (seis) Metros de Tudo Soldável PVC de 32 mm (R\$ 50,00)

1 (um) Cap de PVC para Esgoto Soldável de 75 mm (R\$ 10,00)

6 (seis) Joelhos de 90 Soldáveis em PVC de 32 mm (R\$ 20,00)

7 (sete) Joelhos de 90ㅇ Soldáveis em PVC de 20 mm (R\$ 10,00)

7 (sete) Joelhos de 90 Soldáveis em PVC de 25 mm (R\$ 10,00)

1 (um) Registro de Esfera em PVC Soldável de 20 mm (R\$ 10,00)

1 (uma) Bomba Submersível com Vazão de 2500 L/h (R\$ 250,00)

7 (sete) Flanges em PVC Soldável com Anel de $20 \mathrm{~mm}(\mathrm{R} \$ 70,00)$

2 (dois) Flanges em PVC Soldável com Anel de 25 mm (R\$20,00)

30 (trinta) Parafusos 7/16" X 2" com Porcas e Arruelas $(R \$ 10,00)$

6 (seis) Flanges em PVC Soldável com Anel de 32 mm (R\$ 150,00)

1 (um) Quilo de Tinta Esmalte Sintética na Cor Alumínio (R\$ 30,00)

2 (dois) Registros de Esfera em PVC Soldáveis de 32 mm ( $R \$ 30,00)$

1 (um) Filtro canister biológico externo com ultra violeta ( $\mathrm{R} \$ 559,93$ )

1 (um) Quilo de Tinta Esmalte Sintética na Cor Preta Fosca (R\$30,00)

5 (cinco) Metros de Ripas em Massaranduba $5 \mathrm{~cm} \mathrm{X} \mathrm{3,0} \mathrm{cm} \mathrm{(R \$} \mathrm{20,00)}$

1 (um) Pacote de Presilhas Plásticas para Fixação com 15 cm $(R \$ 15,00)$ 
4 (quatro) Buchas para Redução Soldáveis Curtas de 32x25 mm (R\$ 10,00) 42 (quarenta e dois) Tijolos de Cimento $19 \mathrm{~cm}$ X $19 \mathrm{~cm}$ X $49 \mathrm{~cm}$ (R\$ 140,00) 1 (uma) Extensão de Energia Elétrica de 5 Metros com 4 Tomadas (R\$ 40,00) 6 (seis) Fotosoquetes Microcontrolados Sensor Luz Temporizado (R\$ 120,00) 10 (dez) Placas de Isopor $100 \mathrm{~cm} \mathrm{X} 120 \mathrm{~cm}$ com Espessura 3,0 cm (R\$100,00) 5 (cinco) Metros Quadrados Tela Tipo Sombrite Transparência de 70\% (R\$20,00) 2 (dois) Adaptadores Soldáveis Curtos Bolsa Rosca Registro de 25 mm (R\$ 10,00) 5 (cinco) Adaptadores Soldáveis Curtos Bolsa Rosca Registro de 32 mm (R\$30,00) 1 (um) Pincel de Cerdas Brancas de 21/2" utilizar verniz resinas sintéticas (R\$ 20,00) 1/2 (meio) Metro Cúbico Areia Lavada para os Berçários de Germinação (R\$ 50,00) 1/2 (meio) Metro Cúbico Brita para Construção Número 2 Meios Cultura $(R \$ 50,00)$ 2 (dois) Tubos Adesivos Plásticos para Conexões de PVC Rígido com 75 g (R\$ 30,00) 1 (um) Balde de Plástico de 40 Litros para funcionar como Mineralizador ( $R \$ 40,00$ ) 5 (cinco) Metros de Mangueira Trançada Transparente em PVC de 25 mm $(R \$ 30,00)$ 10 (metros) Metros Mangueira em Silicone para Compressor de Aquário ( $R \$ 20,00)$ 2 (duas) Caixas Plásticas de Preparo da Massa para as Bandejas de Cultivo $(\mathrm{R} \$ 30,00)$ 6 (seis) Lâmpadas LED 10w Tipo 5 Led Grow Cultivo Hidropônico Indoor (R\$ 420,00) 1 (um) Pincel Cerdas Grids de 21/2" para tintas látex, PVA acrílica base água (R\$20,00) 1 (um) Pincel Cerdas Pretas de 21/2" esmaltes sintético óleo verniz e zarcão (R\$ 20,00)

INVESTIMENTO PARCIAL EM HARDWARE $(R \$ 500,00)$ INVESTIMENTO PARCIAL EM BOMBAS E FILTROS (R\$ $1.255,93)$ INVESTIMENTO PARCIAL EM TUBOS E CONEXÕES (R\$ 2.840,00) INVESTIMENTO TOTAL PLATAFORMA DE AQUAPONIA DOMICILIAR (R\$ 4,595.93) 


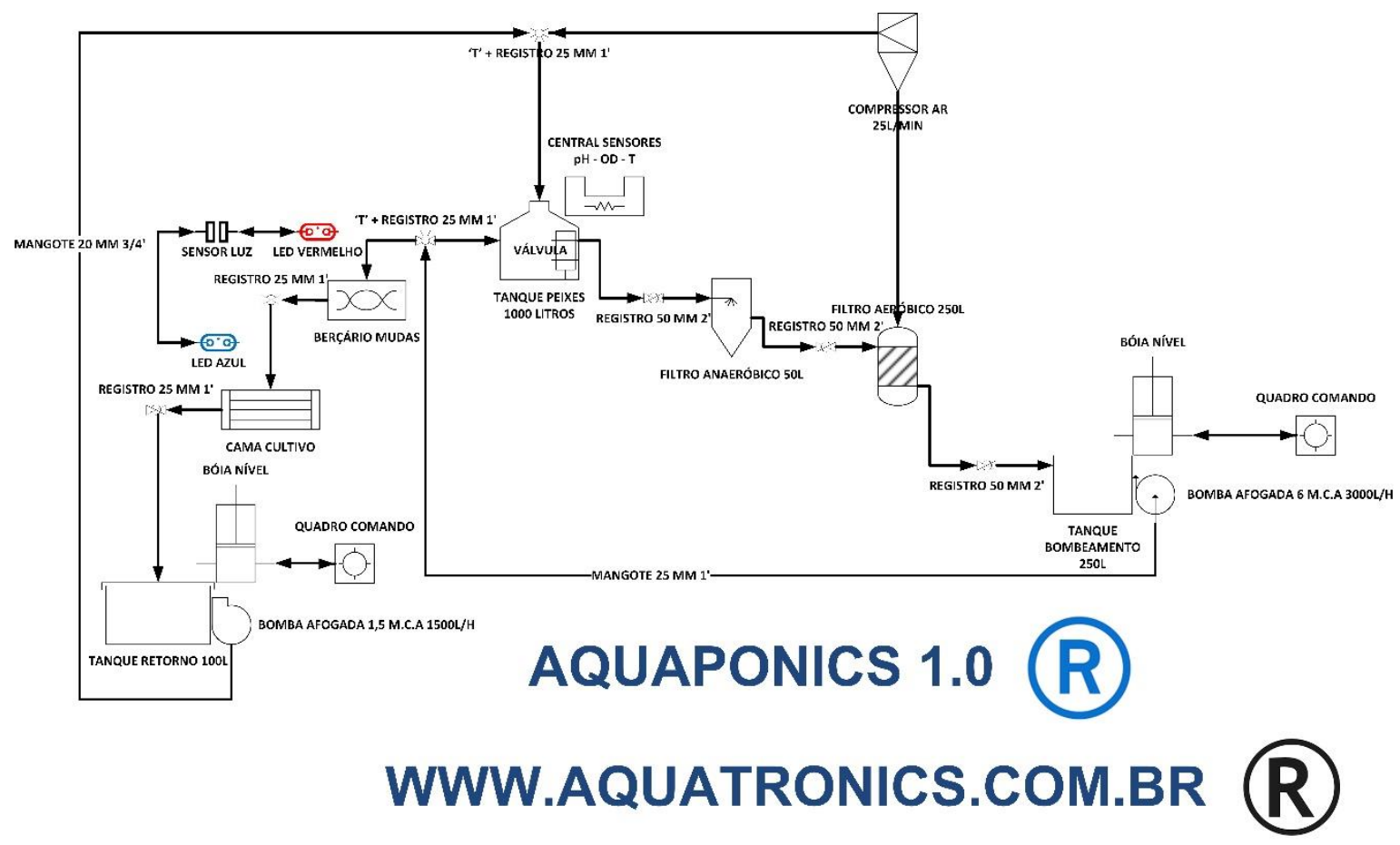

Figura 7 - Esquema Vertical Plataforma Aquaponics 1.0

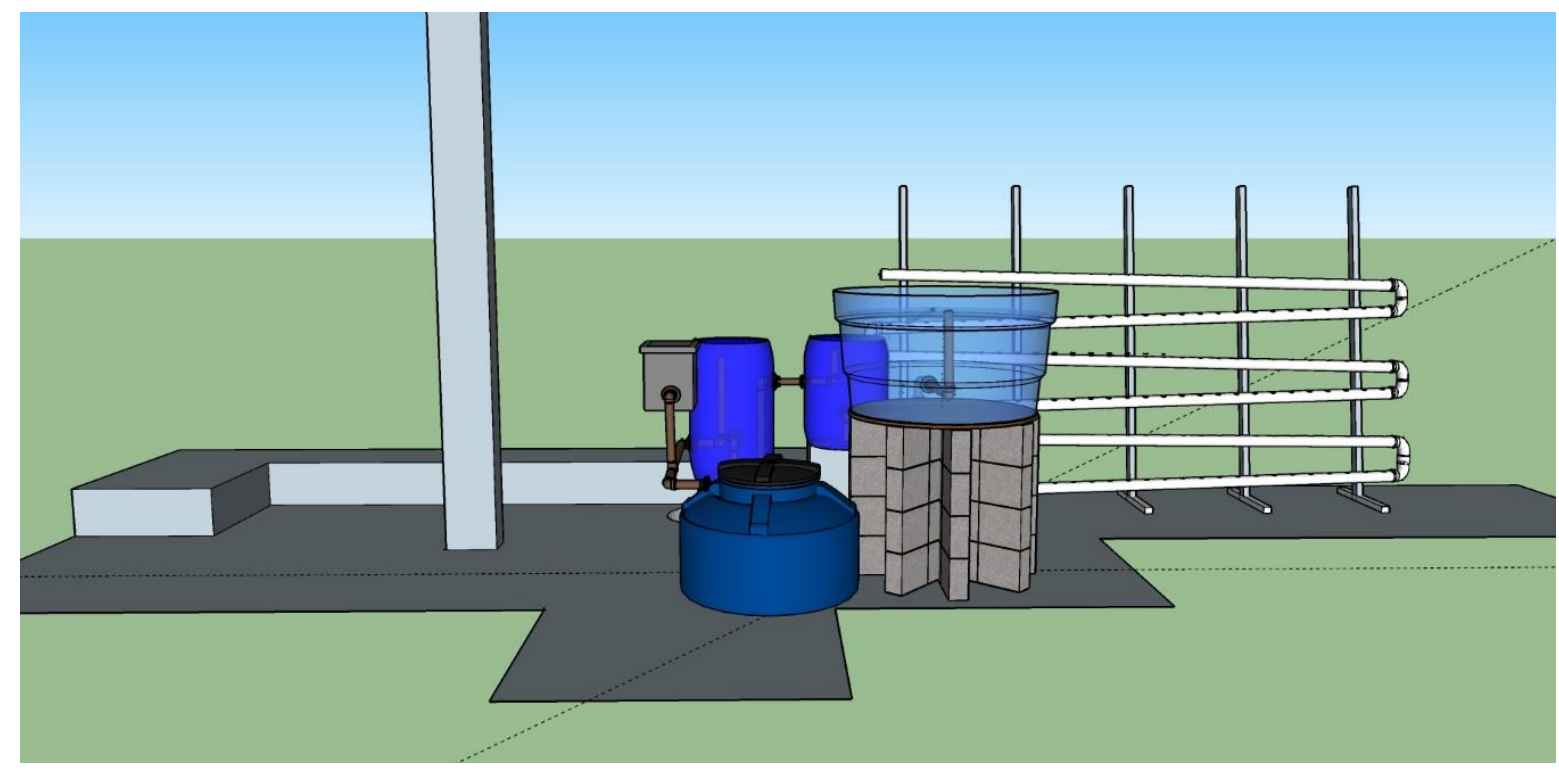

Figura 8 - Projeto 3D Google ScketchUp Plataforma Aquaponics 1.0. 


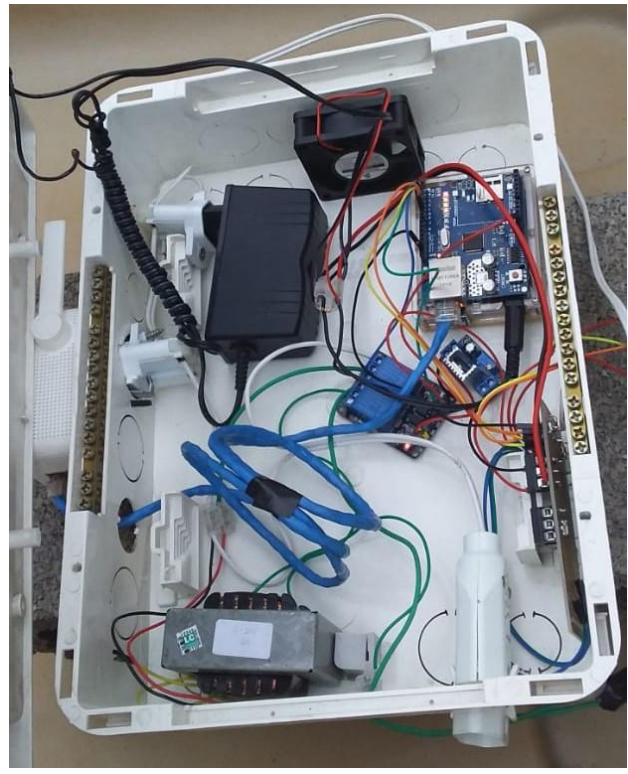

Figura 9 - Central de Comando e Controle Aquaponics 2.0. 


\section{CONSIDERAÇÕES FINAIS}

Após o fechamento da pesquisa foi possível construir uma plataforma operacional com controle do nível da água local automatizado para produção de proteína orgânica em regime domiciliar com um custo inferior a $\mathrm{R} \$ 5.000,00$.

Foi demostrada a viabilidade econômica do projeto para utilização pela classe média brasileira interessada em um estilo de vida alimentar mais natural sem o uso de esteróideas e anabolizantes em animais aquáticos ou inseticidas e agrotóxicos em vegetais hidropônicos.

A utilização da placa microcontroladora no Arduino Uno deixou o sistema extremamente estável dependendo apenas de uma alimentação de energia elétrica contínua. A instalação de um Nobreak poderá aumentar a autonomia do sistema em caso de interrupção acidental do abastecimento por parte da concessionária pública do serviço local.

Os próximos passos da pesquisa estão relacionados a automação dos outros módulos da plataforma incluindo: alimentador temporizado dos peixes, potencial de hidrogênio, condutividade elétrica, potencial de oxidação-redução, nível de oxigênio dissolvido, temperatura, luminosidade e umidade relativa do ar.

Finalmente o código fonte em $\mathrm{C}++$ da automação do controle do nível de água da plataforma está documentado no APÊNDICE A. 


\section{REFERÊNCIAS}

SANTOS, Bruno P., Lucas A. M. Silva, Clayson S. F. S. Celes, João B. Borges Neto, Bruna S. Peres, Marcos Augusto

M. Vieira, Luiz Filipe M. Vieira, Olga N. Goussevskaia e Antonio A. F. Loureiro, Internet das Coisas: da Teoria à Prática, Departamento de Ciência da Computação, Universidade Federal de Minas Gerais (UFMG), Belo Horizonte, MG, Brasil, 2017. Disponível em <http://homepages.dcc.ufmg.br/ mmvieira/cc/papers/internet-das-coisas.pdf>, acessado no dia 27 de abril de 2018.

CASTRO, E. J. R. et al. Engenharia de Requisitos: Um Enfoque Prático na Construção de Software Orientado ao Negócio. Florianópolis, SC: Bookess, 2014.

EMBRAPA, Brasil. Calagem e Controle da Acidez dos Viveiros de Aqüicultura. Circular Técnica 14. ISSN 1516-4683. Jaguariúna, SP: Dezembro, 2006.

Recomendações Práticas para Melhorar a Qualidade da Água e dos Efluentes dos Viveiros de Aqüicultura. Circular Técnica 12. ISSN 1516-683. Jaguariúna, SP: Dezembro, 2006.

. Boas Práticas de Manejo (BPMs) para Reduzir o Acúmulo de Amônia em

Viveiros de Aqüicultura. Comunicado Técnico 44. ISSN 1516-8638. Jaguariúna, SP: Dezembro, 2007.

. O Uso do Sulfato de Cobre em Ecossistemas Aquáticos: fatores que afetam sua toxicidade em peixes de água doce. Documentos 91. ISSN 1516-4691. Jaguariúna, SP: Junho, 2013.

- Montagem e Operação de um Sistema Familiar de Aquaponia para Produção de Peixes e Hortaliças. Circular Técnica 72. ISSN 1678-1945. Aracaju, SE: Dezembro, 2015. 
. Produção Integrada de Peixes e Vegetais em Aquaponia. ISSN 16781953189. Aracaju, SE: Outubro, 2015.

. Sistema Familiar de Aquaponia em Canaletas. Circular Técnica 81. ISSN 1678-1945. Aracaju, SE: Dezembro, 2016.

Loureiro, A. A., Nogueira, J. M. S., Ruiz, L. B., Mini, R. A. d. F., Nakamura, E. F., and Figueiredo, C. M. S. (2003). Redes de Sensores Sem Fio. In Simpósio Brasileiro de Redes de Computadores (SBRC), pages 179-226.

PATTILLO, ALLEN. An Overview of Aquaponic Systems: Hydroponic Components. Technical Bulletin \#123. NCRAC, lowa. March 2017.

An Overview of Aquaponic Systems: Aquaculture Components. Technical Bulletin \#124. NCRAC, lowa. October 2017.

PMI. Um Guia do Conhecimento em Gerenciamento de Projetos. Guia PMBOK ${ }^{\circledR}{ }^{a}$ Edição. Pennsylvania, USA. Project Management Institute, 2013.

Ruiz, L. B., Correia, L. H. A., Vieira, L. F. M., Macedo, D. F., Nakamura, E. F., Figueiredo, C. M., Vieira, M. A. M., Bechelane, E. H., Camara, D., Loureiro, A. A., et al. (2004). Arquiteturas para redes de sensores sem fio.

WAZLAWICK, Raul. Metodologia de Pesquisa para Ciência da Computação. Elsevier, Campus 6a Reimpressão. Rio de Janeiro, 2009. 


\section{APÊNDICE A - CÓDIGO FONTE APLICAÇÃO C++}

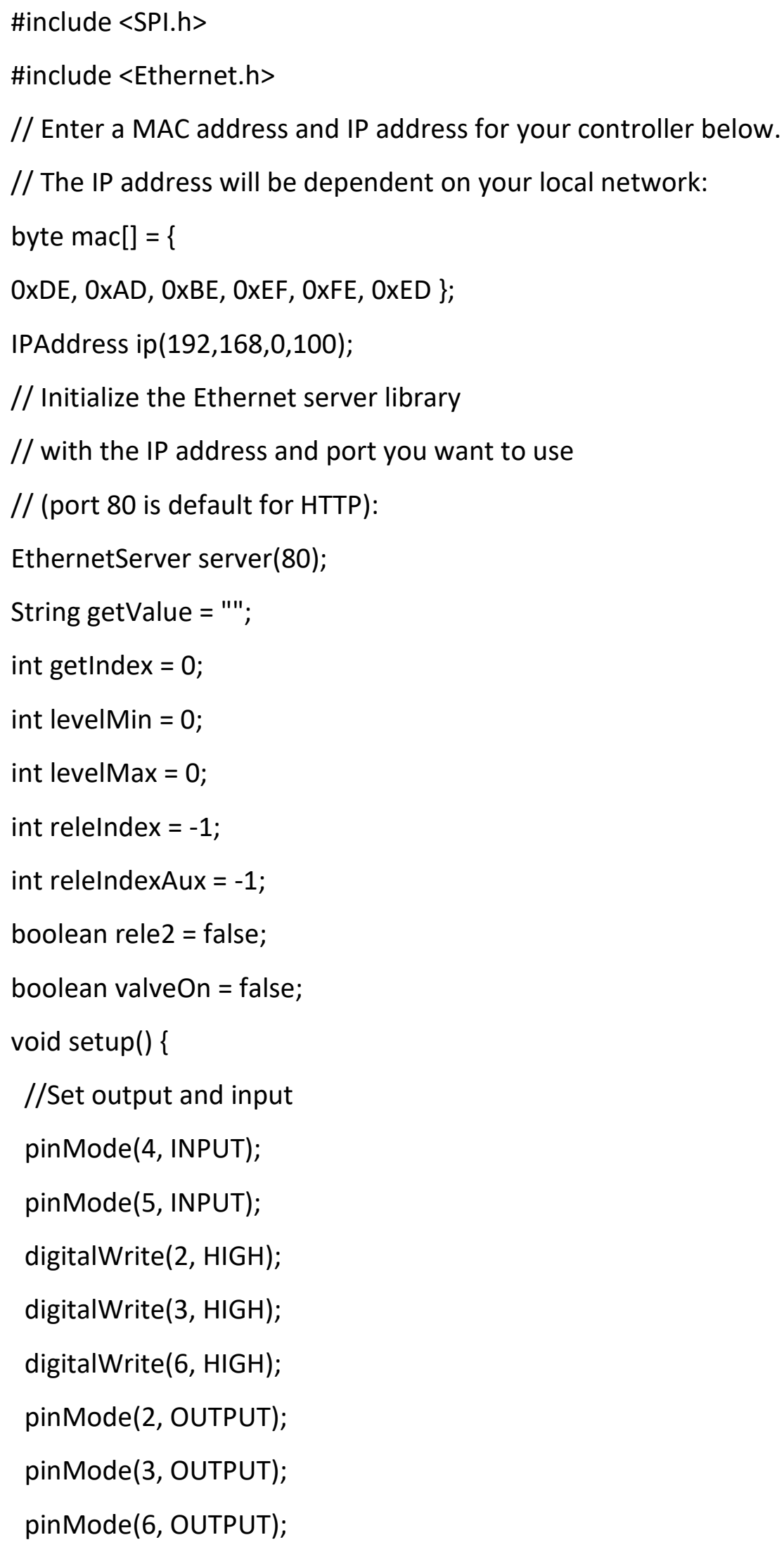


// Open serial communications and wait for port to open:

Serial.begin(9600);

while (!Serial) \{

; // wait for serial port to connect \}

// start the Ethernet connection and the server:

//Ethernet.begin(mac, ip);

Ethernet.begin(mac);

server.begin();

//Serial.print("server is at ");

Serial.println(Ethernet.localIP());

//Serial.printIn(Ethernet.linkStatus());

//Serial.printIn(Ethernet.hardwareStatus()); \}

void loop() \{

//read level sensors

levelMin = digitalRead(4);

levelMax = digitalRead(5);

// listen for incoming clients

EthernetClient client $=$ server.available( $)$;

if (client) \{

Serial.println("new client");

// an http request ends with a blank line

boolean currentLinelsBlank = true;

while (client.connected ()$)\{$

if (client.available()) \{

char c = client.read () ;

if(getValue.indexOf("HTTP")<0) getValue +=c;

Serial.write(c);

//client.print In(c);

// if you've gotten to the end of the line (received a newline

// character) and the line is blank, the http request has ended,

// so you can send a reply

if (c == ' $\backslash n$ ' \& \& currentLinelsBlank) \{ 
// send a standard http response header

client.printIn("HTTP/1.1200 OK");

client.printIn("Content-Type: text/html");

client.printIn("Access-Control-Allow-Origin: *");

client.printIn("Connection: close"); // the connection will be closed after completion of the response

//client.printIn("Refresh: 3"); // refresh the page automatically every $5 \mathrm{sec}$

client.println();

client.printIn("<!DOCTYPE HTML>");

client.print $\ln ("<\mathrm{html}>")$;

//show sensors states

client.print("i;");//client.print("levelMin:");

client.print(levelMin);

client.print(";");//levelMax:");

client.print(levelMax);

//control rele state from http GET command

getIndex = getValue.indexOf("rele");

relelndexAux = getValue.substring(getIndex+5, getIndex+6).tolnt();

if(relelndexAux != 0) relelndex = relelndexAux;

client.print(";");//relelndex:");

client.print(relelndex);

//show valve state

client.print(";");//valve:");

client.print(valveOn);

client.print In("</html>");

//aditional info

getValue = "";

break; \}

if $(c==' \backslash n ')\{$

// you're starting a new line

currentLinelsBlank = true; $\}$

else if $\left(c !=' \backslash r^{\prime}\right)\{$ 
// you've gotten a character on the current line

currentLinelsBlank = false; $\}\}\}$

// give the web browser time to receive the data

delay(1);

// close the connection:

client.stop();

Serial.printIn("client disonnected"); \}

//relelndex reference: 1->both OFF | 2->rele1 ON rele2 OFF | 3->rele1 OFF rele2

ON | 4->both ON

//check sensor level states

if $($ levelMin==LOW) valveOn $=\mathrm{HIGH}$;

if $($ levelMax==HIGH) valveOn = LOW;

valveOn $=$ valveOn || relelndex==2 || relelndex==4;

//valve command

if(valveOn) digitalWrite(3, LOW);

else digitalWrite(3, HIGH);

//led test

if(valveOn) digitalWrite(6, HIGH);

else digitalWrite(6, LOW);

//check index to set the second rele

rele2 $=$ relelndex $==3$ || relelndex $==4$;

//control the second rele via http

if(rele2) digitalWrite(2, LOW);

else digitalWrite(2, HIGH);

\}//end loop 